\title{
Study of Some Power Influencing Parameters of a Solar Low Temperature Stirling Engine
}

\author{
H. El Hassani ${ }^{1}$, N. Boutammachte ${ }^{2}$, M. Hannaoui ${ }^{1}$
}

\begin{abstract}
The aim of this paper is to study experimentally and theoretically the effect of some geometrical parameters on low temperature differential Stirling engines (LT-SE) performance. The studied parameters are: the phase angle, the compression ratio and the dead volume. Results show that for optimizing the performance of these engines, dead volume should be minimized, the compression ratio should be maximized and the optimal phase angle for the gamma type is $90^{\circ}$. For the adopted theoretical model, based on Schmidt theory, even if theoretical numerical results are different from those found experimentally, but it still remains a valid model for finding out some parameters effect on the LT-SE performance, and for calculating approximately the engine work and power.
\end{abstract}

Key words: Stirling engine, low temperature, Solar, Phase angle, compression ratio, dead volume.

\section{Introduction}

The Stirling engine is an externally heated machine; it was invented in 1816 by the Stirling brothers (Kolin, 1991). This engine is a reversible machine and has theoretically Carnot efficiency. Besides, it could use several energy sources, such as solar radiations, which makes this engine, if enough developed a clean solution for energy production.

In 1983, Kolin (Senft, 2004) demonstrated the first low temperature Stirling engine. In the last decades, many researches were interested in this kind of machines. Thus, many prototypes were constructed and tested. Senft (Senft, 2004) (Robson, Grassie, \& Kubie 2007) developed the Ringbom engine using Kolin conclusions. Kongragool and Wongwises (Kongtrarool \& Wongwises 2007) constructed twin power pistons and four power pistons, gamma-configuration LT-SE. Cinar and Karabulut constructed and tested a gamma type LT-SE, this machine was tested with air and helium; its maximum power was $12.3 \mathrm{~W}$.

In 1871, G. Schmidt proposed a simple second order analysis of the ideal Stirling engine cycle (Schmidt, 1871). The Schmidt analysis has been used widely as an approximation of the Stirling engine power calculation, and Berchowitz and Urieli (Urieli \& Berchowitz, 1984) gave a detailed description of it.

As a result of a scientific cooperation between Moulay Ismail University in Morocco, and the Technological University of Dresden in Germany, a gamma type LT-SE was constructed and tested. Thus, this paper aims to study theoretically and experimentally 
this prototype. It aims especially to find out the effect of some parameters on this engine performance, and on LT-SE performance in general. The studied parameters are: the phase angle, the compression ratio and the dead volume. The experimental study was conducted in order to find out the real effect of some of the former parameters on the engine power, and to eventually valid the theoretical model adopted.

\section{Prototype description}

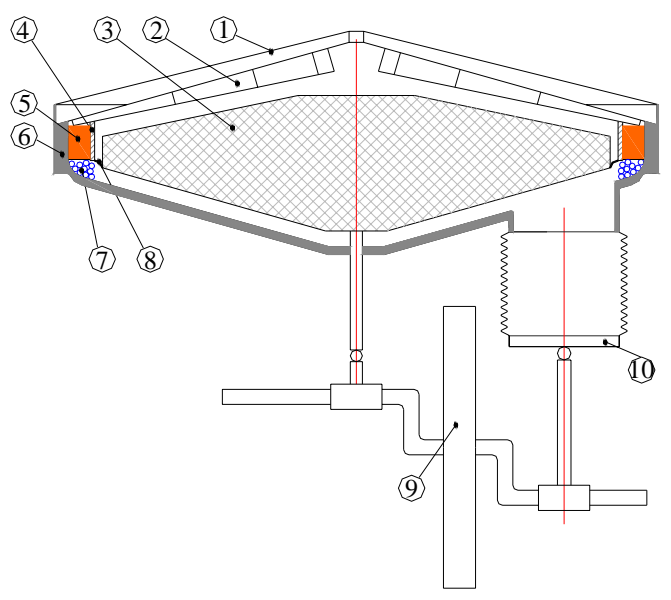

$1:$ Cover

2: Absorber

3 : Displacer

4 : Cylinder

$5:$ Regenerator

6 : Body

7 : Cooler

8 : Membrane

9: Flywheel

10 :Working

piston

Fig.1. Schematic illustration of the studied machine

\begin{tabular}{|c|c|}
\hline \multicolumn{2}{|c|}{ Nomenclature } \\
\hline$n$ & Engine speed, $[\mathrm{Hz}]$ \\
\hline$P$ & Pressure, $[\mathrm{Pa}]$ \\
\hline$r$ & Specific gas constant, $[\mathrm{J} / \mathrm{Kg} \mathrm{K}]$ \\
\hline$T$ & Temperature, $[\mathrm{K}]$ \\
\hline$V$ & Volume, $\left[\mathrm{m}^{3}\right]$ \\
\hline$V_{s c}$ & Maximum volume of hot space, $\left[\mathrm{m}^{3}\right]$ \\
\hline$V_{\text {STR }}$ & Maximaum volume of working space, $\left[\mathrm{m}^{3}\right]$ \\
\hline$W$ & Work, []] \\
\hline$a$ & Phase angle, $\left[{ }^{\circ}\right]$ \\
\hline$\Phi$ & Crank angle, $\left[^{\circ}\right]$ \\
\hline$\eta$ & Efficiency, [-] \\
\hline$\varepsilon$ & Compression ratio, [-] \\
\hline \multicolumn{2}{|c|}{ Subscripts } \\
\hline$c$ & Hot space \\
\hline$f$ & Cold space \\
\hline$m$ & Dead (volume) \\
\hline$r$ & Regenerator \\
\hline TR & Working space \\
\hline
\end{tabular}


Fig.1 shows a schematic illustration of the studied engine and Table 1 indicates some of its design parameters. The absorber is made of an aluminum plate, and the displacer cylinder is made of a composite material. For the displacer and the working piston, they are made of polystyrene. Two crank drives connect the working piston and the displacer to the drive shaft, and the phase angle between the pistons is set at $90^{\circ}$. A steel matrix is placed between the cold and the hot chambers and acts as a regenerator.

\section{Engine work and power}

Schmidt (Schmidt, 1871) presented a mathematically exact expression for calculating the indicated work and power of Stirling engines. The Schmidt theory adopts many ideal assumptions, like perfect regeneration, isothermal compression and expansion and harmonic motion of the displacer and the working piston.

Table 1 Some studied engine parameters

\begin{tabular}{ll}
\hline Parameter & Description \\
\hline Engine type & $\gamma$ \\
Cooling system & Water tubes \\
Displacer diameter & $1.4 \mathrm{~m}$ \\
Displacer stroke & $0.08 \mathrm{~m}$ \\
Working piston diameter & $0.25 \mathrm{~m}$ \\
Working piston stroke & $0.2 \mathrm{~m}$ \\
Absorber diameter & $1.6 \mathrm{~m}$ \\
\hline
\end{tabular}

For applying the Schmidt theory to our studied machine, we divide its volume as shown in Fig.2. Schmidt first calculated the gas mass in different parts of the machine, and used the perfect gases equation, thus we obtain the following equation:

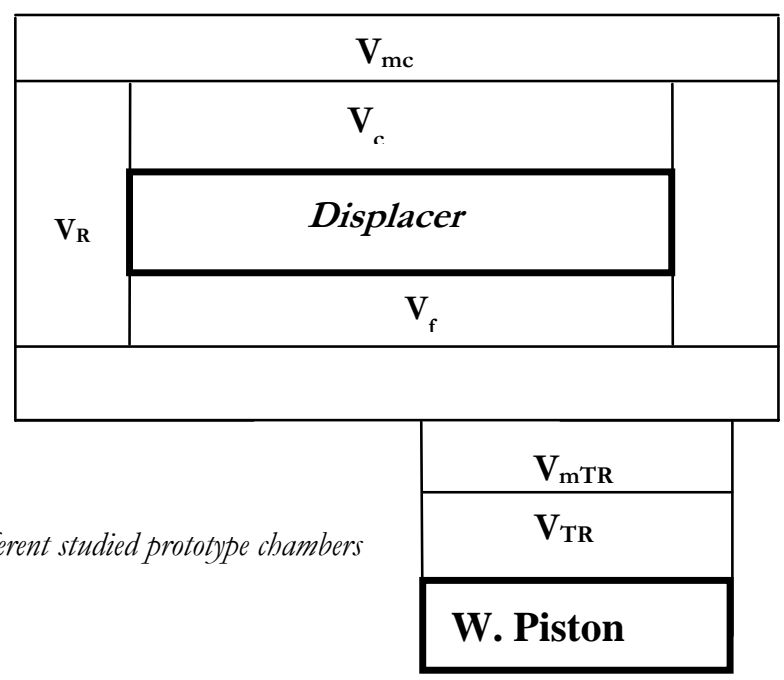




$$
p=m r\left(\frac{V c}{T c}+\frac{V_{f}}{T_{f}}+\frac{V_{T r}}{T_{f}}+\frac{V r}{T r}+\frac{V_{m c}}{T c}+\frac{V_{m f}}{T_{f}}+\frac{V_{m T r}}{T_{f}}\right)^{-1}
$$

Schmidt adopted the assumption of harmonic motion:

$$
\begin{aligned}
& V c=\frac{V S c}{2}(1+\cos (\theta+\alpha)) \\
& V f=\frac{V S C}{2}(1-\cos (\theta+\alpha)) \\
& V_{T R}=\frac{V_{S T R}}{2}(1+\cos \theta)
\end{aligned}
$$

Table 2

Some studied prototype data

\begin{tabular}{ll}
\hline Parameter & value \\
\hline $\mathbf{V}_{\text {mc }}$ & $0.010 \mathrm{~m}^{3}$ \\
$\mathbf{V}_{\mathbf{R}}$ & $0.047 \mathrm{~m}^{3}$ \\
$\mathbf{V}_{\text {mf }}$ & $0.010 \mathrm{~m}^{3}$ \\
$\mathbf{V}_{\text {mTR }}$ & $0.001 \mathrm{~m}^{3}$ \\
Vsc & $0.123 \mathrm{~m}^{3}$ \\
$\mathbf{V}_{\text {sTR }}$ & $0.01 \mathrm{~m}^{3}$ \\
Displacer stroke & $0.08 \mathrm{~m}$ \\
W.Piston stroke & $0.2 \mathrm{~m}$ \\
$\mathbf{T}_{\mathbf{c}}$ & $343 \mathrm{~K}$ \\
$\mathbf{T}_{\mathbf{f}}$ & $293 \mathrm{~K}$ \\
$\mathbf{n}$ & $30 \mathrm{rpm}$ \\
$\boldsymbol{\alpha}$ & $90^{\circ}$ \\
\hline
\end{tabular}

The pressure is expressed as following:

$$
\begin{gathered}
P=\frac{m r / k}{1+\left(\frac{A}{k}\right) \cos (\theta-\beta)} \\
k=\frac{V_{S C}}{2 T c}+\frac{V_{S C}}{2 T f}+\frac{V_{S T R}}{2 T f}+\frac{2 V_{r}}{T f+T c}+\frac{V_{m c}}{T c}+\frac{V_{m f}}{T f}+\frac{V_{m T R}}{T f} \\
A=\frac{1}{2}\left[\left(\frac{V_{S T R}}{T f}\right)^{2}+\left(\frac{V S c}{T c}-\frac{V S c}{T f}\right)^{2}-2 \frac{V_{S T R}}{T f}\left(\frac{1}{T f}-\frac{1}{T c}\right) V S C\right. \\
\quad \times \cos \alpha]^{\frac{1}{2}}
\end{gathered}
$$

Cyclic work and power are expressed as follows:

$W_{\text {tot }}=W_{T R}=\int_{0}^{2 \pi} P d V_{T R}=-\frac{1}{2} V_{S T R} \int_{0}^{2 \pi} P \sin \theta d \theta$ 
$W_{\text {tot }}=V_{S T R} \frac{\pi m R}{A}\left(\frac{1}{\sqrt{1-k_{1}^{2}}}-1\right) \sin \beta$
$P=\frac{n \times W t o t}{2 \pi}=V_{S T R} \frac{n m r}{2 A}\left(\frac{1}{\sqrt{1-k_{1}^{2}}}-1\right) \sin \beta$

Where $n$ is the engine speed and $m$ is the total gas mass.

\section{Theoretical parametric study}

In order to find out the effect of some LT-SE parameters on these engines power, a parametric study based on the theoretical analysis was performed. Thus the effect of the dead volume, the phase angle, and the compression ratio on these engines power was studied. Note that Schmidt calculating assumptions are adopted.

At each study, only one parameter is changed in order to find out its single effect. Results are found by using a computer calculation program. For a complete crank revolution, and at a step of $1^{\circ}$, the program computes the instantaneous positions of the working piston and the displacer, the volume of the machine chambers, the total mass, work and power.

\subsection{Study of the dead volume effect}

In order to find out the effect of the dead volume on LT-SE power, a calculation of the variation of the studied machine power and work according to the dead volume value was performed. The studied engine data are available in Table 2. Results are shown in Fig. 4

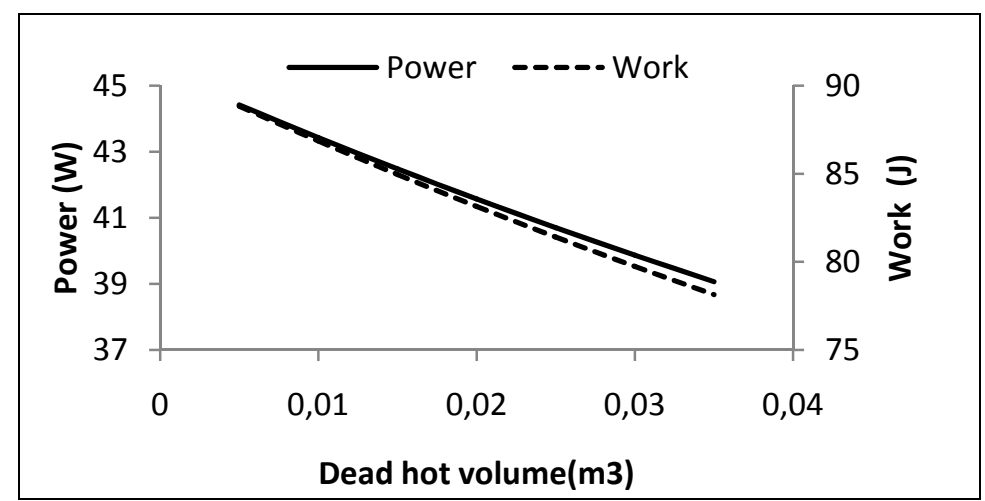

Fig.4. Effect of the dead hot volume on the engine work and power

Fig. 4 indicates the negative effect of the dead hot volume on the power of LT-SE. This result might be explained by the fact that the dead volume is a quantity of gas that does not participate to the thermodynamic cycle even if it consumes heat from the heat source. Moreover, it prevents active gas from reaching the absorber, thus, it forms a gas layer limiting heat transfer to it, which explains its negative effect on power. For the 
same reasons, a similar effect could be expected for all the rest of the engine dead volumes. Thus while designing LT-SE, dead volumes should be minimized.

\subsection{Study of the compression ratio}

For visualizing the effect of the compression ratio on LT-SE performance, a calculation of power was established for many compression ratios. Results for the studied prototype are shown in Fig. 5.

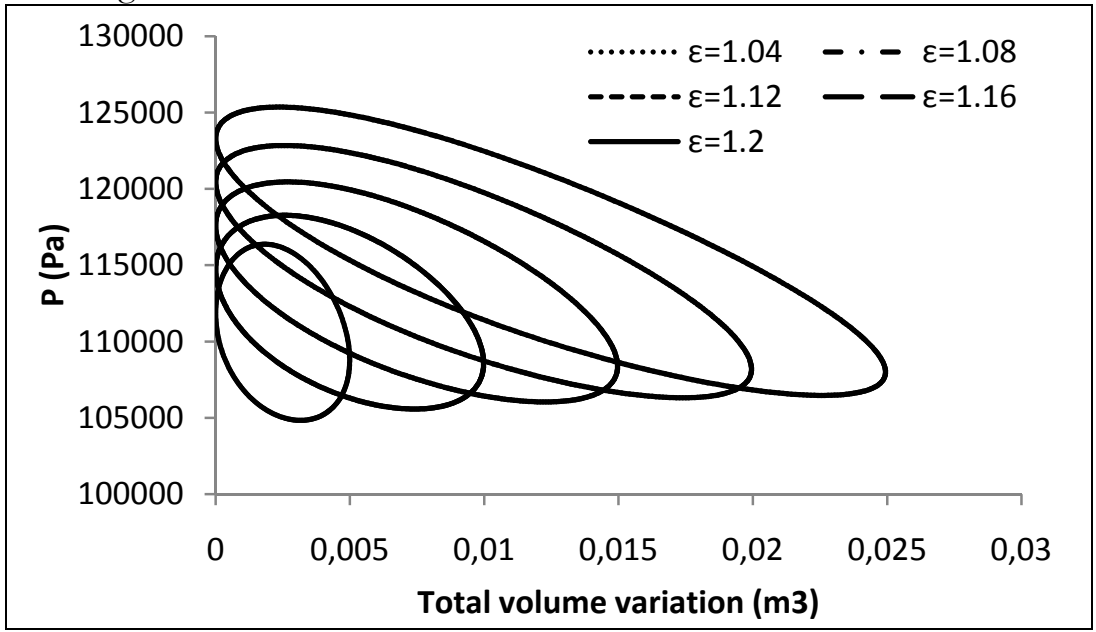

Fig.5. Effect of the compression ratio on the studied machine Clapeyron diagram

Fig. 5 indicates a positive effect of the increasing of the compression ratio value on the studied engine performance. In fact the Clapeyron diagram gets bigger internal surface by the increasing the value of this parameter. In order to quantify this positive effect, a calculation of the indicated work and power was established. Results are shown in Fig. 6.

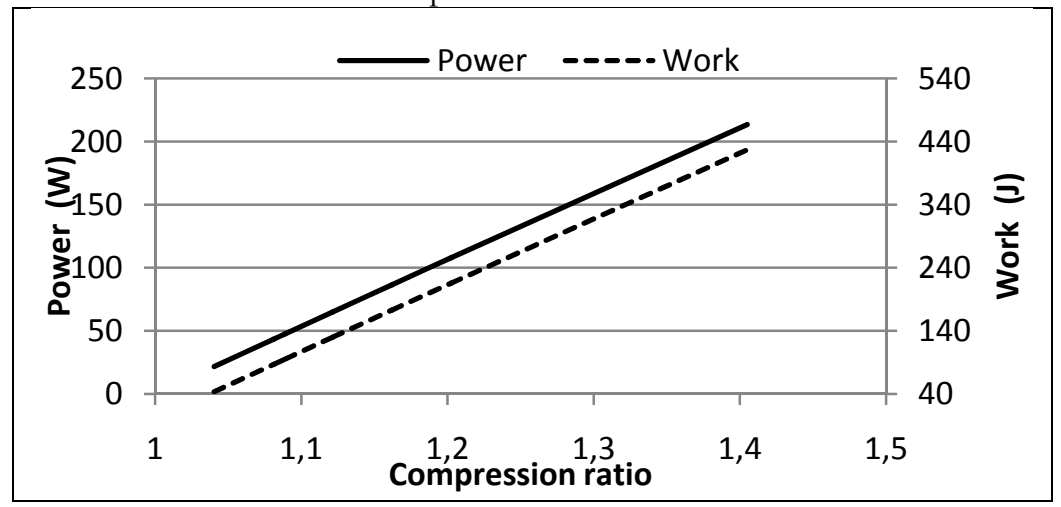

Fig.6. Effect of the compression ratio on work and power

Fig. 6 confirms results of the former figure. In fact, for the studied machine, an increasing of 0.1 in the compression ratio value implies an increasing by $21 \mathrm{~W}$ in the power output, which indicates the importance of this parameter for enhancing the LTSE performance. 


\subsection{Study of the phase angle effect}

In order to find out the effect of the phase angle between the LT-SE pistons on the power output, we draw the Clapeyron diagram for many phase angle values. Results are shown in Fig. 7.

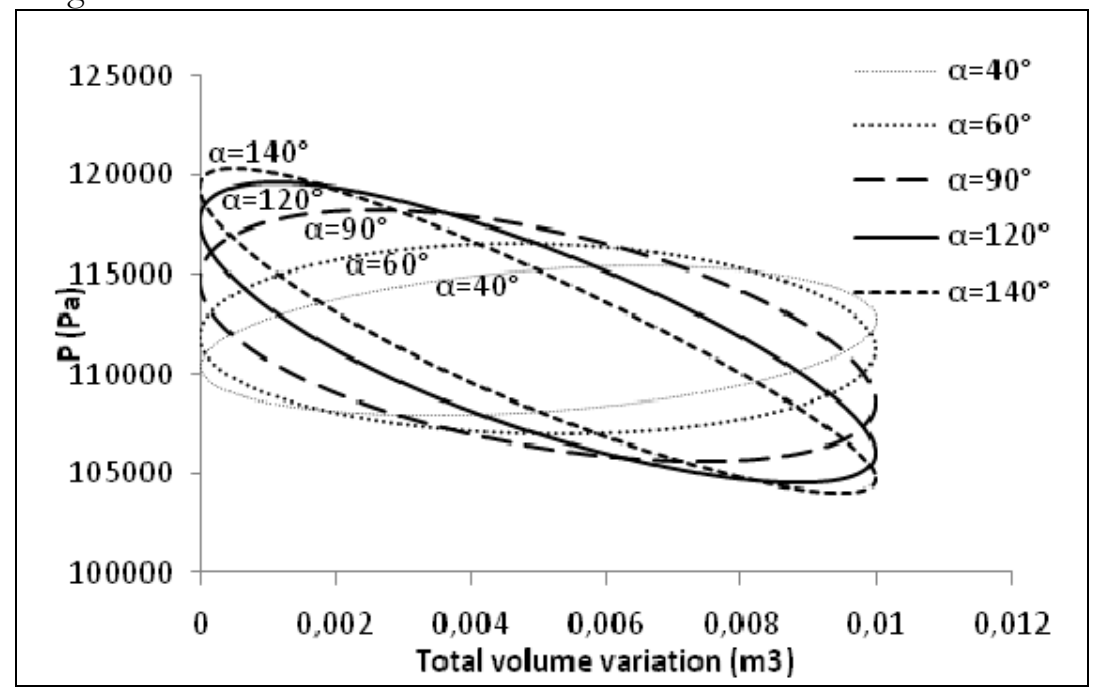

Fig.7. Effect of the phase angle on the studied machine Clapeyron diagram

Fig. 7 indicates that the Clapeyron diagram shape varies according to the phase angle value. In fact, it indicates that more the phase angle is greater; more the Clapeyron diagram shape approaches the theoretical one. We even notice that for the value $40^{\circ}$ of the phase angle, the diagram gets reversed. Thus, in order to approach the ideal Stirling cycle, which gives Carnot efficiency, small phase angle values should be avoided for gamma type LT-SE. In order to find out the optimal phase angle for this kind of machines, we calculated the indicated work and power for many phase angle values. Results are shown in Fig. 8.

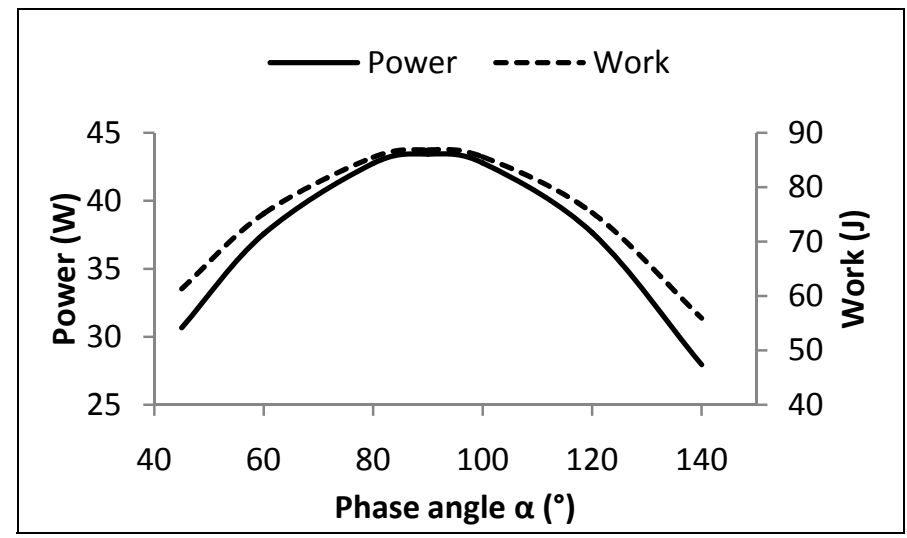

Fig.8. Effect of the phase angle on work and power 
Fig. 8 indicates that the optimal phase angle for gamma type LT-SE is about $90^{\circ}$, thus this phase angle should be adopted while designing of this kind of machines.

\section{Experimental study}

In order to find out the real effect of some of the former studied parameters on LT-SE performance, and valid the adopted calculation model, an experimental study was performed on the studied prototype under real conditions in Morocco. The studied parameters are the phase angle and the compression ratio.

\subsection{Experimental setup}

The following setup was used in order to perform the experimental study:

- $\quad \mathrm{K}$-type thermocouples with $\pm 0.001{ }^{\circ} \mathrm{C}$ accuracy were used in order to measure the temperature inside different prototype chambers.

- A pressure sensor with $\pm 0.1 \mathrm{~Pa}$ accuracy was used in order to measure the pressure inside the machine.

- For identifying the flywheel position for every temperature and pressure measurement, a position sensor was fixed to the drive shaft; its accuracy is $\pm 0.0001^{\circ}$.

- The variation of the phase angle was possible by varying the connection between the engine pistons thanks to the perforation of many holes in the crank drive mechanism.

- The variation of the compression ratio was performed by the variation of the working piston stroke.

\subsection{Experimental effect of the phase angle}

Based on the experimental setup described previously, and for many phase angle values, we draw the Clapeyron diagrams and calculated the indicated work and power. Results are shown in Fig. 10.

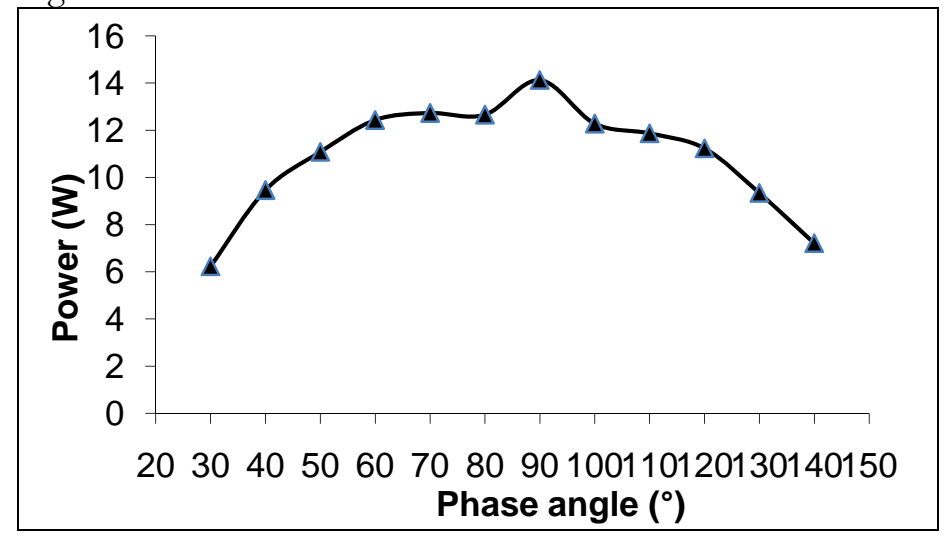

Fig.10. Experimental phase angle effect on power output

Fig. 10 indicates that the optimal phase angle which gives the maximum power ouput is $90^{\circ}$, which is the same result found previously theoretically. However, numeric results 
are different. In fact, for the phase angle $90^{\circ}$, the theoretical power is about $42 \mathrm{~W}$ while the experimental one is about $14 \mathrm{~W}$.

This difference could be explained by the fact that the theoretical adopted model, which is deduced from the Schmidt theory, adopts many assumptions which do not take into consideration some real phenomena, like the imperfect regeneration and the nonisothermal transformations. Besides many technical problems cause many losses, which participate to the decreasing of the engine power, like: charge loss due to the regenerator, pressure leakage, heat loss in the displacer and in the walls, etc.

However, despite the noticed numerical difference between theoretical and experimental results, the adopted model is still a valid one for finding out the effect of these engines parameters on their performance, and approach the power real value.

Finally, as a conclusion for the studied parameter, the phase angle $90^{\circ}$ should be adopted while designing the gamma type LT-SE in order to maximize the indicated power.

\subsection{Experimental effect of the compression ratio}

In order to find out experimentally the effect of the compression ratio on the LT-SE performance, we varied this parameter value by varying the working piston stroke, and drawn the corresponding Clapeyron diagrams. Fig.11 shows the results.

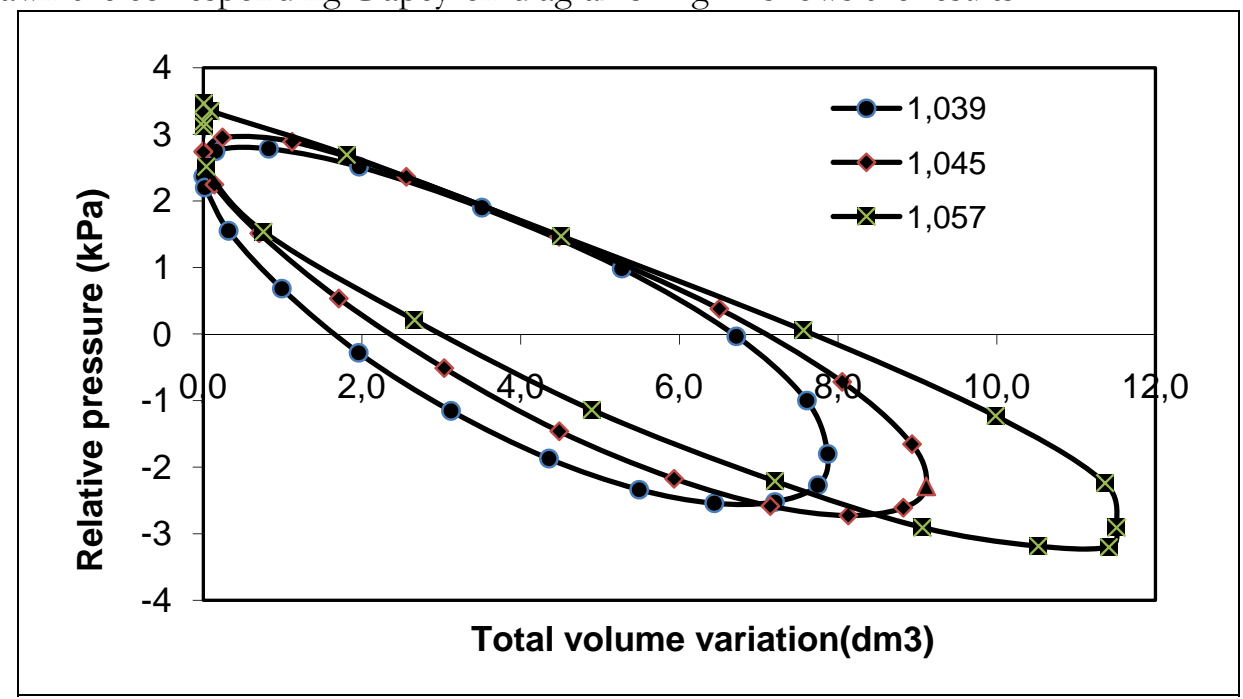

Fig.11. Experimental effect of the compression ratio on the Clapeyron diagram of the studied machine

Fig. 11 confirms the theoretical results found previously in the theoretical study. In fact, Fig. 11 shows that the indicated work increases with the increasing of the compression ratio. However, as noticed for the parameter phase angle, theoretical and experimental numerical values are different. In fact, for the compression ratio $\varepsilon \approx 1.04$, theoretical results indicate a power value about $21,75 \mathrm{~W}$, while experimental results indicate a value about $11.14 \mathrm{~W}$. Similarly as done for the parameter phase angle, we can explain this noticed difference by the same reasons, and deduce the same conclusion relatively to the adopted model. 
Thus, experimental and theoretical results indicate that the increasing of the compression ratio increases the LT-SE power. This result can be explained by the fact that the increasing of this parameter value makes the maximum pressure during the compression stage getting greater, or/and the minimum pressure during the expansion stage becoming smaller, which has a positive effect on the engine work. The Clapeyron diagram also becomes taller.

However, the compression ratio could not be increased infinitely; its maximum value depends on the engine volume, since the working piston stroke is limited by the engine geometry. Besides, by increasing the pressure inside the engine, the leakage becomes a considerable problem which should be taken into consideration, especially if the engine cost parameter is an important one.

\section{Conclusion}

In this paper a gamma type low temperature Stirling engine was studied theoretically and experimentally. The effect of the dead volume, the phase angle and the compression ratio on this machine work and power was analyzed. Results showed that for optimizing this engine performance, and the LT-SE in general, dead volume, should be minimized, the compression ratio should be maximized as the machine allows it, and the optimal phase angle for the gamma type is $90^{\circ}$. For the adopted theoretical model, based on Schmidt theory, even if numerical results that it gives are different from those found experimentally, but it still remains a valid model for finding out some parameters effect on the LT-SE performance, and for calculating approximately the engine work and power.

\section{References}

Kolin, I. (1991). Stirling Motor: History-theory-practice. Zagreb: Zagreb University . Kontragool, B. \& Wongwises, S. (2007). Performance of low-temperature differential Stirling engines. Renew Energ, 32, 547-566

Robson, A. ,Grassie, T. , \& Kubie, J. (2007). Modeling of a low-temperature differential Stirling engine. Proc IMechE Part C J Mech Eng Sci, 221,927-943

Schmidt, G. (1871). Theorie der lehmannschen calorischen maschine. Zeit. des Vereines deutsch. Ing., 15, 1-12

Schmidt, G. (1871). Theorie der lehmannschen calorischen maschine. Zeit. des Vereines deutsch. Ing., 15, 97-112.

Senft, JR. (2004). An Introduction to low temperature differential Stirling engines. USA: Moriya Press.

Urieli, I. \& Berchowitz, D. (1984). Stirling cycle engine analysis, Alternative sources of energy. Bristol: Adam Hilger. p.71. 